\title{
A HISTOCHEMICAL STUDY OF PULMONARY HYALINE MEMBRANE
}

\author{
BY \\ F. DURAN-JORDA, A. HOLZEL and W. H. PATTERSON \\ From Booth Hall Children's Hospital, Manchester \\ (RECEIVED FOR PUBLICATION NOVEMBER 22, 1955)
}

At necropsy on premature infants, microscopic examination of the lungs often reveals the presence of eosinophilic material which forms a hyaline membrane in the alveoli and bronchioles. There seems little doubt of the close association between the intense respiratory distress observed clinically and the presence of this membrane at necropsy. However, there is, as yet, no general agreement on the pathogenesis of the condition, and the controversy is more than academic for it seems that a rational therapeutic approach must await a satisfactory explanation of its origin.

The relevant literature on hyaline membrane up to 1952 has been reviewed by Tran-Dinh-De and Anderson (1953). Broadly, there are two main schools of thought. First, the advocates of aspiration of amniotic fluid ('exogenous aetiology'), though at present in the majority, appear to be losing ground. Secondly, there are those who consider that the membrane is formed in alveoli and bronchioles in situ and that it is the result of vascular injury. Increasing evidence in support of this concept is appearing in the literature. There are a few workers who call on factors, both exogenous (aspiration) and endogenous (exudative), in an attempt to produce an acceptable thesis (Stevenson and Laufe, 1955).

Having studied the methods adopted hitherto to find out the basic structure of hyaline membrane it was considered that it would be possible and rewarding to pursue this fundamental aspect further.
A strong bias towards the endogenous theory on clinical grounds was a purposeful stimulus along a difficult road which, however, already had some signposts for which earlier workers must be thanked. This paper is, therefore, an account of some avenues explored in an attempt to discover the nature of hyaline membrane.

Morphological studies by Gilmer and Hand (1954) led them to postulate an endogenous origin for the eosinophilic material primarily from the blood stream, and they considered also that an additional possibility was an alteration of the basement membrane to form the hyaline substance or, alternatively, the formation of it between capillary and pulmonary basement membrane. Lynch and Mellor (1955) concluded from their histological studies that 'hyaline membranes' represent a highly concentrated secretion from the epithelial cells of the terminal bronchioles and alveolar ducts.

It seemed more profitable to study the hyaline membrane of infants in comparison with eosinophilic material occasionally found in pulmonary oedema or in other parts of the body such as in thrombosed vessels, or in the albuminous precipitate surrounding some glomeruli of the kidney. By the use of a whole battery of various staining techniques it was possible to demonstrate that the different precipitates behaved, from that aspect, in a similar manner (Table 1). The results of the methods employed suggested strongly that the hyaline membrane

TABLE 1

STAINING REACTIONS

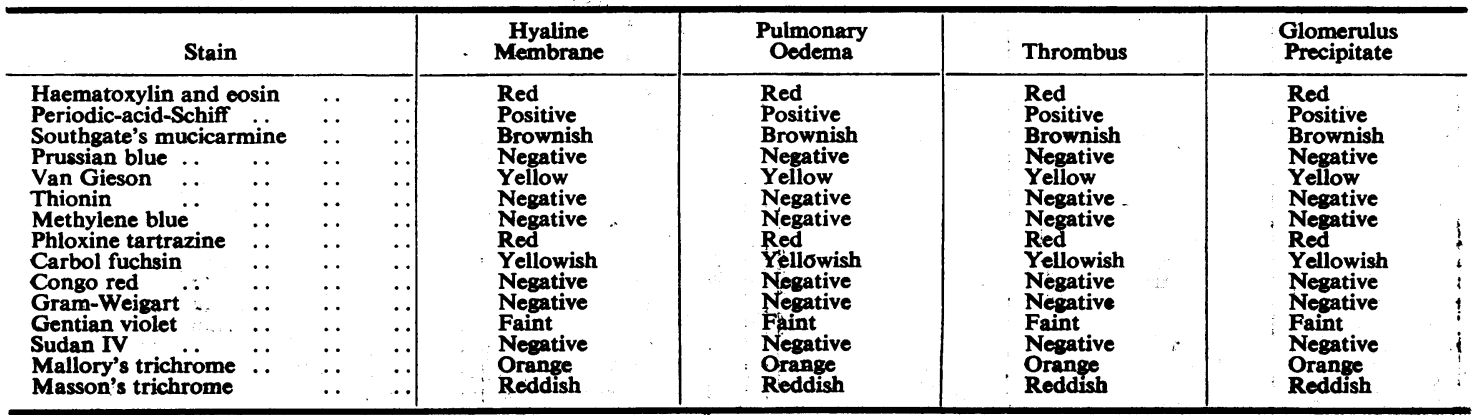




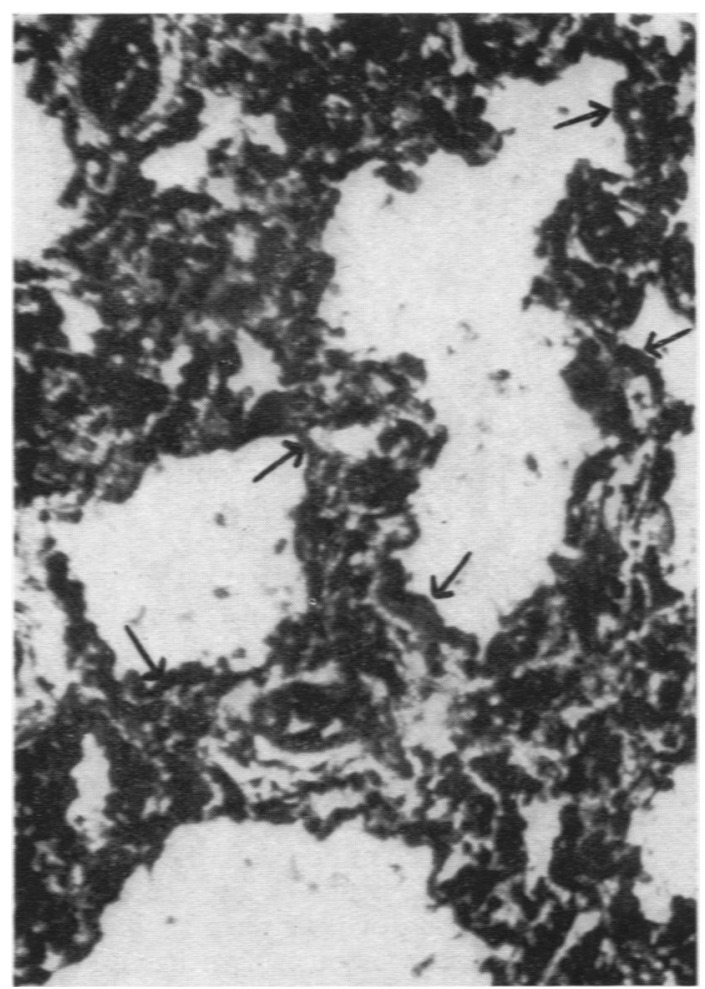

Fig. 1.- Lung of a recently born baby containing hyaline membrane stained with the Pickworth technique. The hyaline membrane gives a positive reaction.

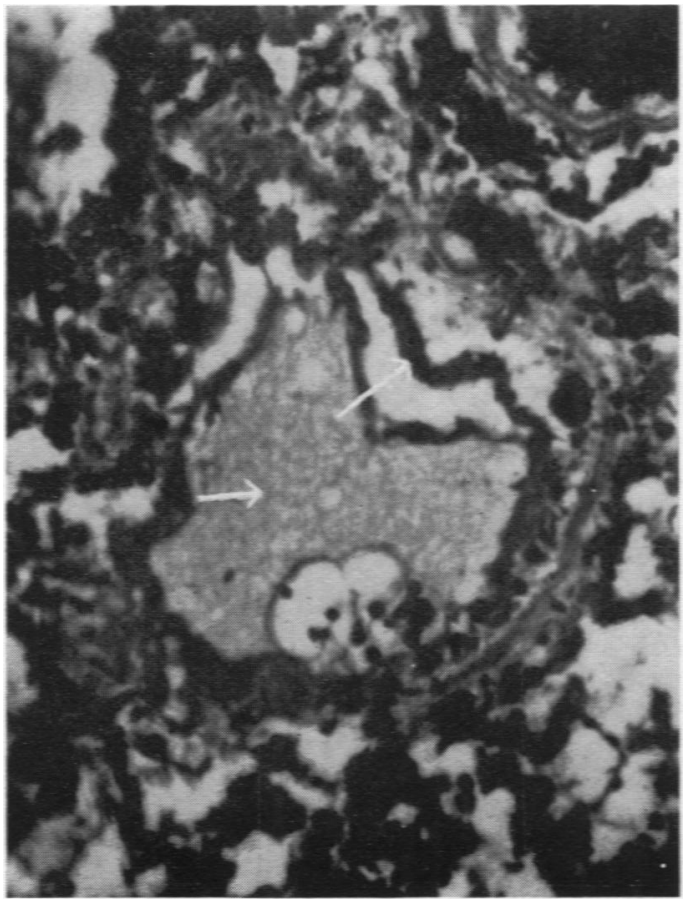

Fig. 2.-Pulmonary oedema stained with Pickworth technique. The exudate gives a positive reaction.

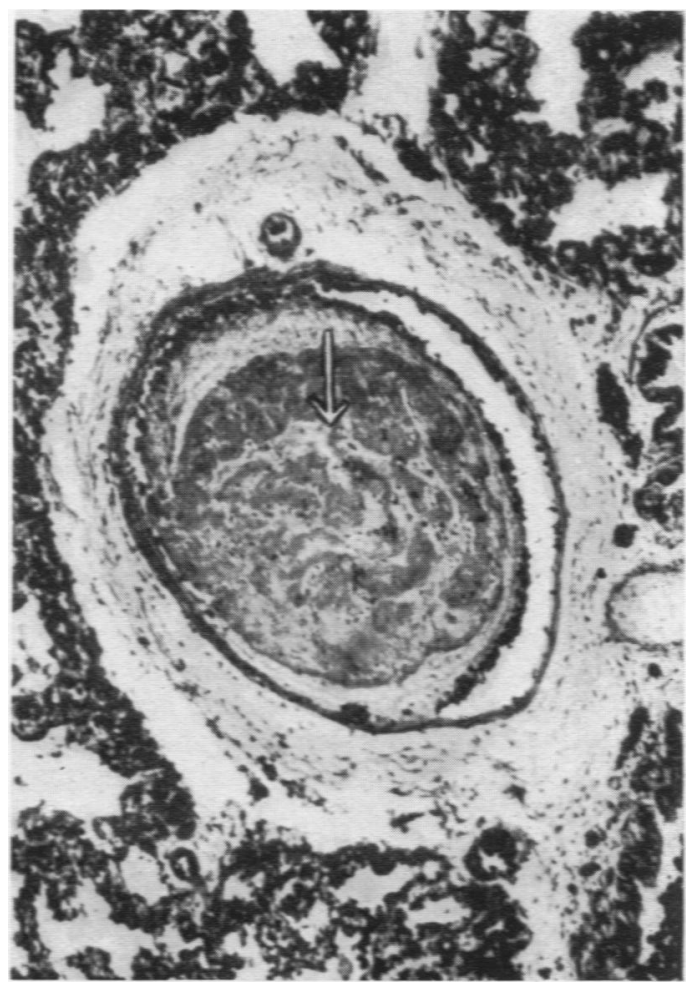

Fig. 3.-Acellular material found in a lung vessel thrombus stained with the Pickworth technique. The intravascular material gives a positive reaction.

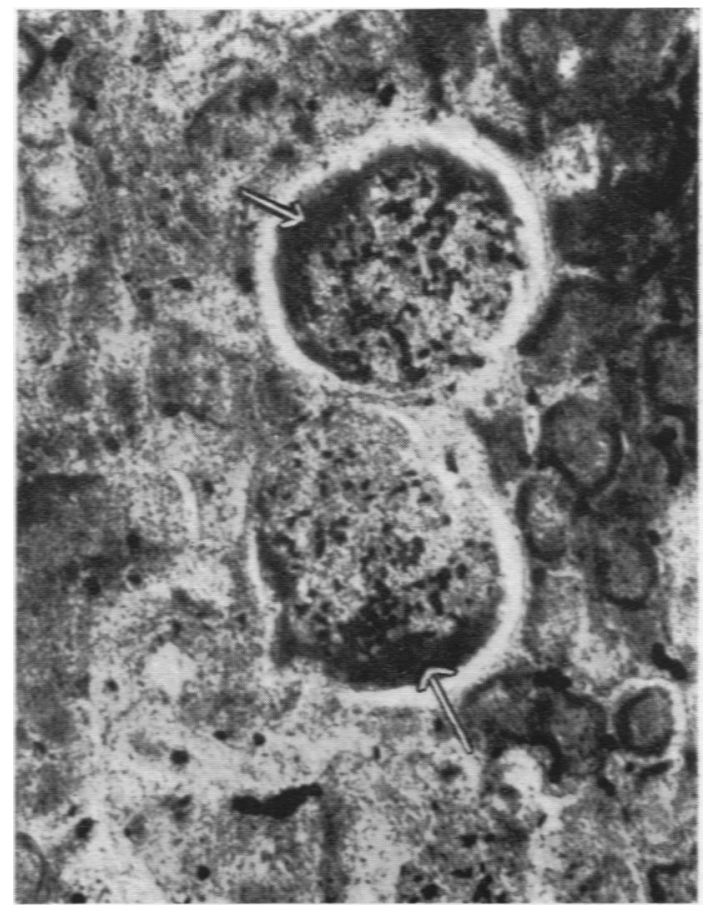

Fig. 4.-Exudate round a renal glomerulus stained with the Pickworth technique. The exudate gives a positive reaction.

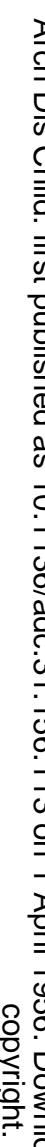

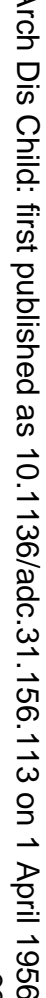

응

을

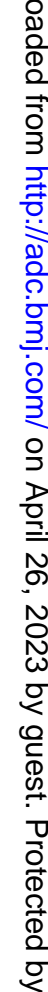




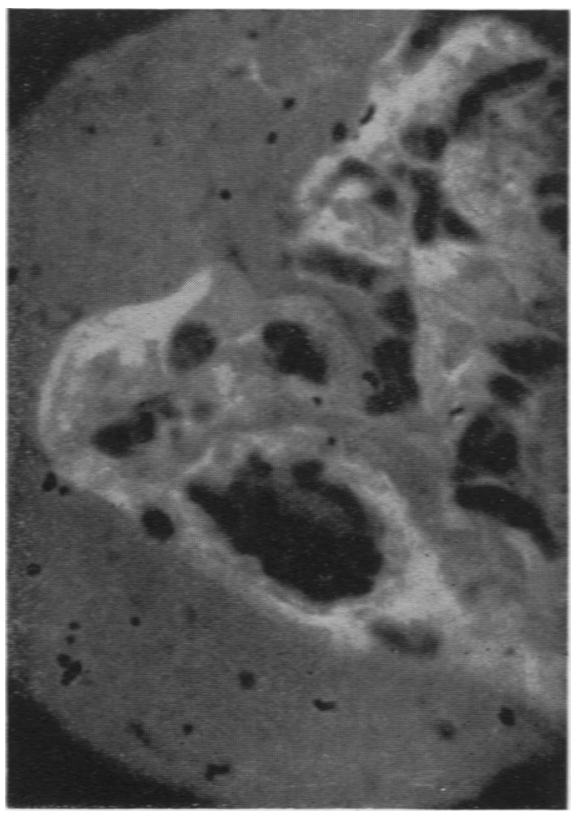

FK. 5.-Fluorescence of hyaline membrane using Wood's light, green filter and auramine.

Fig. 6.-Continuous spectrogram of A, blank: B, red blood cells: $C$, hyaline membrane. The similarity can be seen between $B$ and $C$.

FiG. 7.-Mercury vapour light spectrograms of A, blank: B, red blood cells: C, hyaline membrane. The similarity between $B$ and $C$ can again be seen.

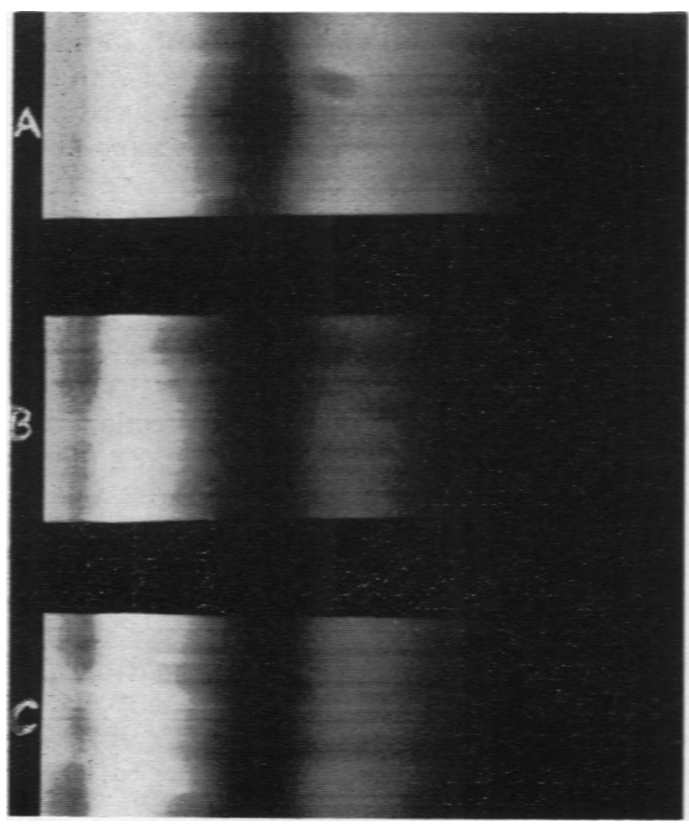

belonged to the group of cytochromes derived from blood proteins and pigments and, therefore, the Pickworth technique (as described in Pearse, 1953) commended itself for further study. All the materials examined gave a positive peroxidase reaction* (Figs. $1,2,3,4)$. In view of these results attention was directed to other techniques employed in the investigation of blood derivatives. Under ultra-violet light using a Wood's filter (Wagner, 1950), the hyaline membrane showed a faint but definite primary fluorescence. On staining the relevant sections of lung with auramine the hyaline membrane showed, as expected, this phenomenon much more clearly (Fig. 5).

To test further the relationship between hyaline membrane and the haemoglobin group, a capillary containing blood and a blank control giving the absorption of lenses and glass slides were submitted to microspectrography using a technique described in a previous communication (Ardern and DuranJorda, 1953). Using a Tungsten light the three spectrograms were taken on the same photographic plate. The hyaline membrane showed absorption in the same region of the violet band as the blood in the capillary vessel (Fig. 6).

* The results of work using this technique were demonstrated to the Section of Pathology of the Manchester Medical Society. 


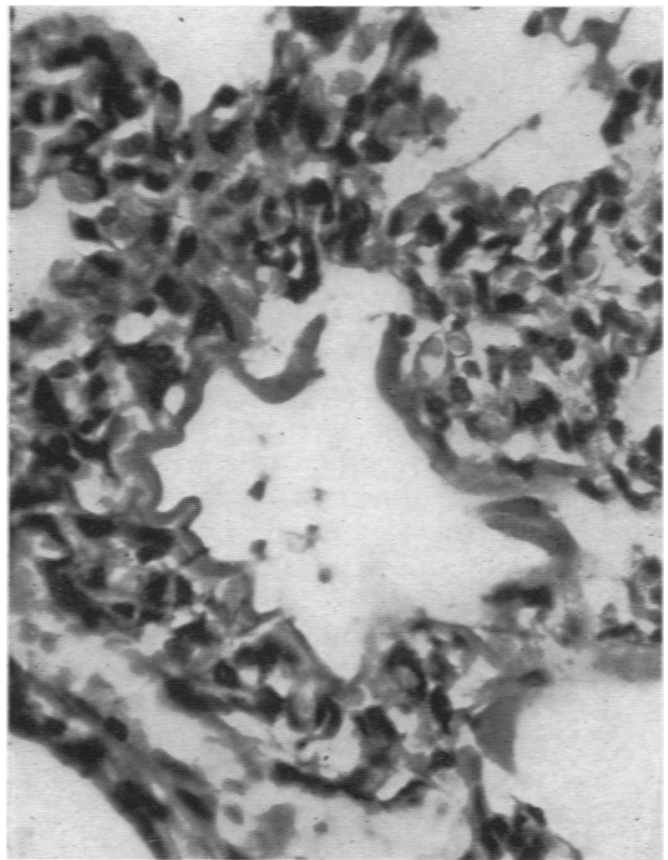

Fig. 8.-Section of lung with hyaline membrane stained with haematoxylin and eosin.

In order to confirm this finding the absorption of the hyaline membrane was studied under a mercury

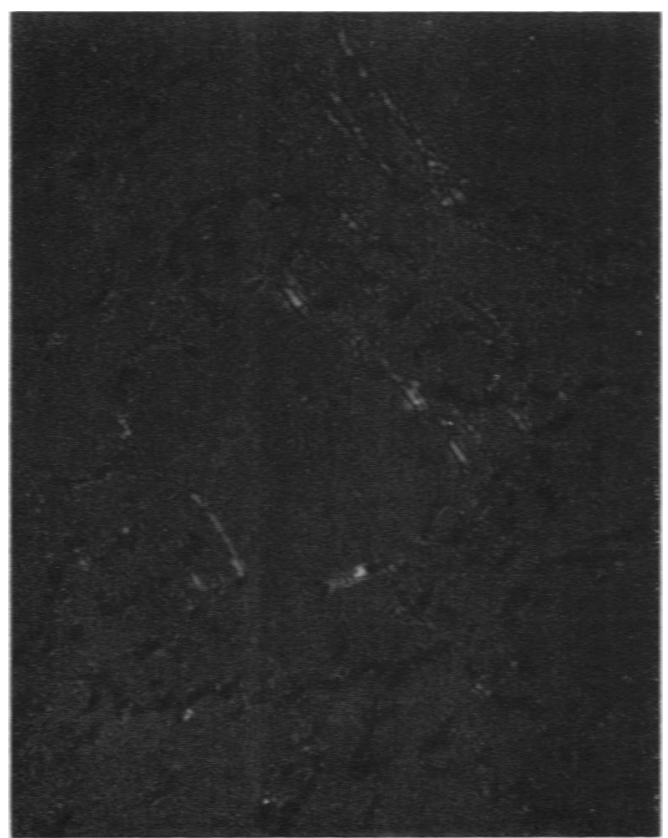

Fig. 10.- The same micro-incinerated field under polariscopic light showing the silica contained in the hyaline membrane.

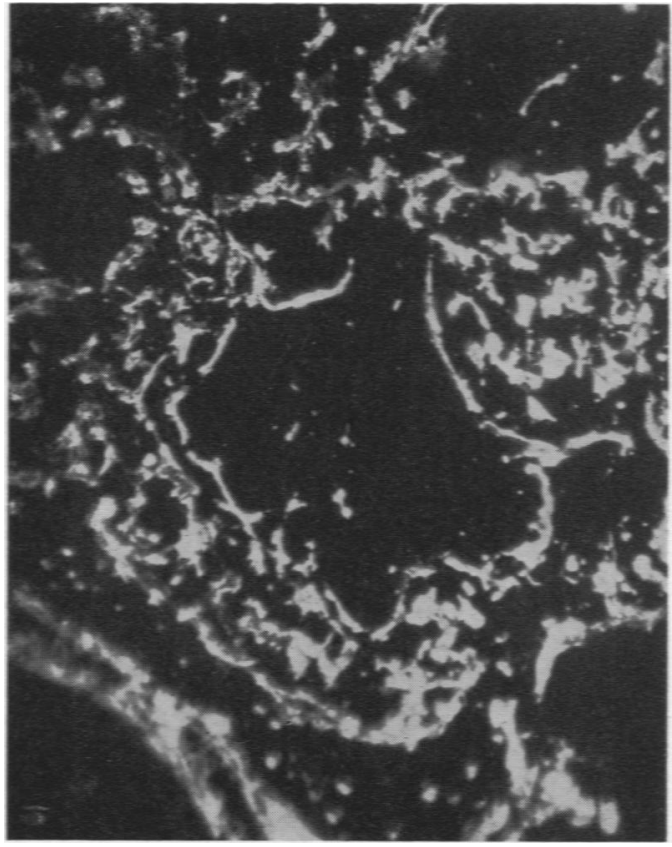

FIG. 9.-The same field after micro-incineration demonstrating the ashes left by the hyaline membrane.

vapour lamp, as its light produces four prominent lines of absorption in a fixed part of the spectrum. Comparing again the spectrogram of hyaline membrane and blood in a capillary from the same section in the fourth band (the area in which blood pigments show maximal absorption), an almost identical spectrographic pattern was obtained. As with the Tungsten light a blank control was introduced and the three spectra photographed on the same plate (Fig. 7).

As an additional step to explore the basic structure of the hyaline-like material and, at the same time, to eliminate the possibility of its consisting of fat or gluco-proteins (mucus), sections of the lung with hyaline membrane were submitted to the drastic process of microincineration (Scott, 1933). A selected microscopic field of the same section was photographed and then incinerated (Fig. 8). Subsequently, the same field was examined with a dark condenser and a second photograph taken. It could be easily observed that the hyaline membrane left a considerable amount of ashes (Fig. 9). Some of these were red, indicating the presence of iron salts, and examination under polarized light showed also a silica content (Fig. 10).

At this stage it was decided to study the material remaining after dialysing amniotic fluid to determine if there was any hyaline substance. Polythene 


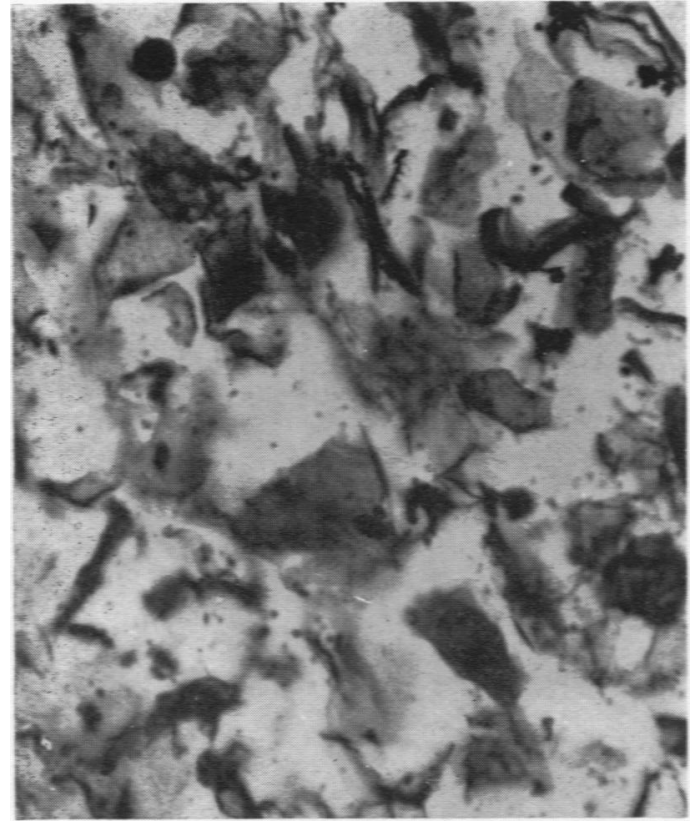

FrG. 11.-Section of amniotic fluid remnants after dialysis showing epithelial cells and debris, but no eosinophilic material.

tubing was used and a $10^{\circ}{ }_{0}$ solution of dextran as dialysing fluid. The residue, a firm white substance, was collected, fixed in formol saline and embedded in paraffin. After sectioning and staining the remnants were seen to be composed entirely of epithelial cells. No eosinophilic material could be found (Fig. 11).

\section{Discussion}

The work described was undertaken because it was felt that the continued belief that hyaline membrane was the result of inhalation of amniotic fluid was not contributing to an understanding of its pathogenesis and so far had not led to a satisfactory therapeutic approach.

The basic fact is that while the membrane is found mainly in newborn premature babies it has been found in the lungs outside the neonatal age group in a number of conditions. For example, exposure of the lungs to high oxygen concentrations in aviation has resulted in the formation of the membrane (Ingalls, 1954). In some instances such hyperoxia has produced a combination of haemorrhagic pneumonia and membrane formation. This finding is of particular interest because of the clinical impression that there is a close association between the so-called haemorrhagic pneumonia of the newborn and hyaline membrane disease (Fig. 12). Often it would seem to be merely a matter of degree.
Lendrum (1955) postulates that the pulmonary hyaline membrane is a manifestation of left heart failure in the newborn premature infant. He feels that the familiar terminology as at present used is objectionable because 'it centres attention upon a single and possibly minor member of a highly constant group of manifestations'.

It was not the purpose of this paper to claim that hyaline membrane disease is a clinical entity. Sufficient evidence is available to suggest that histology reveals an end-result rather than a cause. It is claimed, however, that the evidence produced here weighs heavily in favour of an endogenous origin for pulmonary hyaline membrane, and that it is a blood derivative. This being so it would seem to follow logically that the present therapeutic effort of trying 'to dissolve the membrane' by medicated mists, etc., is not the proper line of approach, and appears about as futile as trying to cure faucial diphtheria by scraping the membrane off the throat.

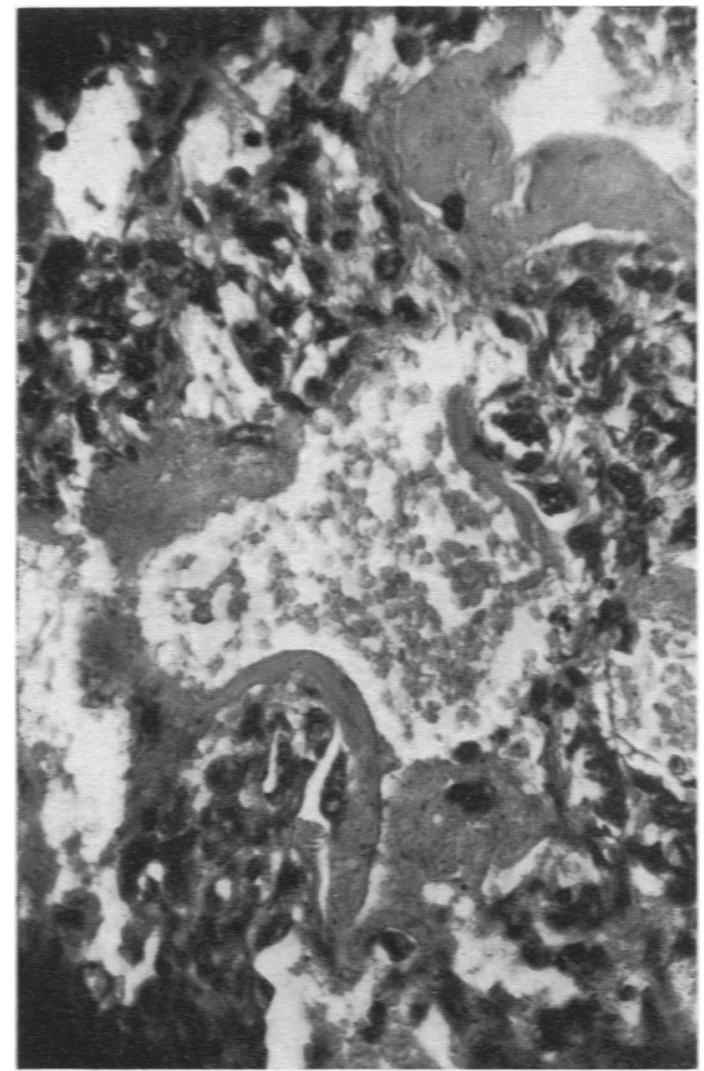

FK. 12.-Section of haemorrhagic newborn lung with a very thick hyaline membrane. 


\section{Summary}

A planned series of laboratory investigations to determine the nature of hyaline membrane is described. It is concluded from these investigations that the membrane is derived from the lung capillaries. Its nature suggests that it follows intense congestion of the alveolar capillaries which result in an exudative process through the capillary walls. It seems to be an end-result rather than a cause, and capable of explanation without postulating aspiration of amniotic fluid. The complete absence of hyaline material from amniotic fluid is noted.

Our thanks are due to Drs. Crawford and Langley for supplying some of the pathological material and to Dr. Bevis for help with the work on amniotic fluid. Mr. T. Walsh's painstaking technical assistance was much appreciated. The Hospital Management Committee made a generous grant from the hospital's research fund.

\section{REFERENCES}

Ardern, F. W. and Duran-Jorda, F. (1953). American Symposium of Spectrography, May, 1953. Gilmer, W. S. and Hand, A. M. (1955). Arch. Path. (Chicago),

Ingalls, T. H. (1954). New Engl. J. Med., 251, 1017.

Lendrum. F. C. (1955). J. Pediat. 47, 149.

Lynch, M. J. G. and Mellor, L. D. (1955). Ibid., 47, 275.

Pearse, A. G. E. (1953). Histochemistry. London.

Scott, G. H. (1933). Amer. J. Anat., 53, 243.

Stevenson, S. S. and Laufe, L. E. (1955). J. Pediat., 47, 40.

Tran-Dinh-De and Anderson, G. W. (1953). Obstet. gynec. Surv., $8,1$.

Wagner, K. (1950). Wien. med. Wschr., 100, 808. 